\title{
BEAM DYNAMICS SIMULATIONS FOR A DC GUN BASED INJECTOR FOR PERL*
}

\author{
F.Zhou ${ }^{1,2, \dagger}$, I.Ben-Zvi ${ }^{2}$, X.J.Wang ${ }^{2}$, \\ ${ }^{1}$ UCLA, Los Angeles, CA 90095, USA \\ 2 Brookhaven National Laboratory, Upton, NY 11973, USA
}

\begin{abstract}
The National Synchrotron Light Source (NSLS) at Brookhaven National Laboratory (BNL) is considering an upgrade based on the Photoinjected Energy Recovering Linac (PERL). The various injector schemes for this machine are being extensively investigated at BNL. One of the possible options is photocathode DC gun. The schematic layout of a PERL DC gun based injector and its preliminary beam dynamics are presented in this paper. The transverse and longitudinal emittance of photoelectron beam were optimized for a DC field $500 \mathrm{kV}$.
\end{abstract}

\section{INTRODUCTION}

The proposed PERL based X-ray light source [1] at the NSLS of Brookhaven National Laboratory (BNL) will take advantage of high-brightness electron beam source and highly efficient energy recovery from superconducting linac. The flexibility and femto-seconds time structure of PERL light source will open many new frontiers to explore biological and material structures. The performance of the PERL will be determined by the electron source. The major characteristics of the PERL injector are [2]:

- Beam energy of about $25 \mathrm{MeV}$ and energy spread of $0.1 \%$, i.e., $25 \mathrm{keV}$, and bunch length of 3 ps.

- Beam current of $200 \mathrm{~mA}$, i.e., $0.15 \mathrm{nC}$ per bunch for an L-band linac

- Normalized transverse emittance below $1 \mathrm{~mm}$-mrad for a charge $0.15 \mathrm{nC}$ per bunch.

The advantages of a photocathode DC gun are the possibility of a very good vacuum [2], a lower thermal emittance provided by a GaAs cathode and flexible pulse train structure. Electrical breakdown and relative low beam energy in DC gun are major limiting factors in its performance. Both photocathode RF gun [2] and DC gun were extensively investigated at BNL as PERL injector. In the following sections, the schematic layout of DC gun based injector and its beam dynamics simulation are presented.

\section{LAYOUT OF DC GUN BASED INJECTOR}

Fig.1 shows the schematic layout of the phtocathode DC gun considered. It is composed of a $500 \mathrm{kV} \mathrm{DC}$ gun,

\footnotetext{
* Supported US DOE contract Number DE-AC02-98CH10886.

†'zhouf@sun2.bnl.gov
}

two solenoids, $1.3 \mathrm{GHz}$ bunching cavity, and two $1.3 \mathrm{GHz}$ standing wave accelerating structures.

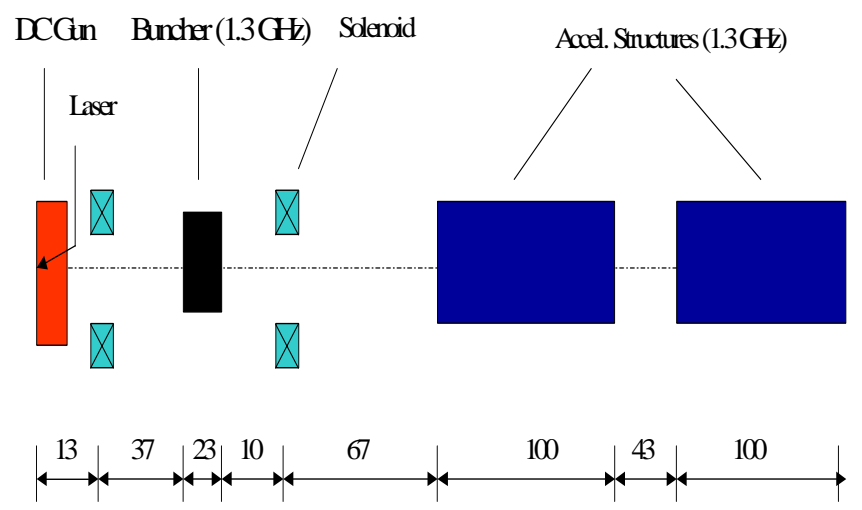

Figure 1: Schematic layout of a DC gun. Dimensions along the beam axis are given in $\mathrm{cm}$.

\subsection{Gun and Solenoids}

The space charge induced emittance growth in the DC gun can be expressed as [3]:

$$
\varepsilon_{n, r m s}=30 \arccos \left(\frac{1}{\gamma}\right) \cdot\left(\frac{I}{E_{0}}\right) \mu_{i}(A),(i=x, z)
$$

where $\gamma$ is the relativistic beam energy factor at the gun exit, $I=\frac{Q}{\sqrt{2 \pi} \sigma_{t}}$ is the peak current, $\mathrm{Q}$ is the bunch charge, $\sigma_{t}$ is the bunch length, $E_{0}$ is the DC field gradient on the cathode, $A=\frac{\sigma_{x f}}{\sigma_{z f}}$ is the rms aspect ratio of the beam at the DC gun exit, and $\mu_{x}^{-1}(A)=3 A+5$, $\mu_{z}^{-1}(A)=1+4.5 A+2.9 A^{2}$ for gaussian distribution. To minimize the space charge induced emittance, high electric fields at the cathode are necessary. Using the parameters of the CEBAF FEL project [4], $500 \mathrm{kV} \mathrm{DC}$ gun is considered for our initial studies. The geometry of the gun and its electrical field distribution are shown in Figure 2. The electrical field distribution was calculated using the computer program POSSION.

There are two solenoids in the beam line. The first solenoid immediately follows the DC gun is responsible for transverse space charge emittance compensation. Its 

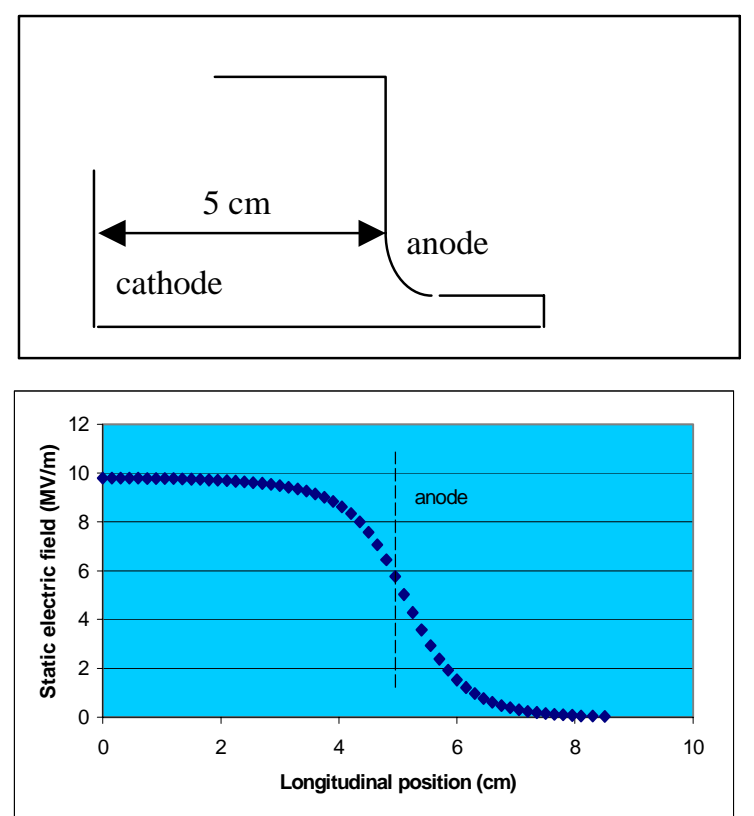

Figure 2: Schematic of DC gun, DC gun Electric field

maximum field is 520 Gauss. The magnetic field on the cathode is kept to zero. The $2^{\text {nd }}$ solenoid is located after the buncher. This solenoid plays two functions; one is to match the beam optics to the down-stream accelerating structures, and the other is for the further emittance compensation due to the low energy after the buncher. The optimized magnetic field is about 550 Gauss.

\subsection{Buncher and Accelerating structures}

The L-band buncher consists of two standing wave cells with a field gradient $7.5 \mathrm{MV} / \mathrm{m}$. The buncher is used to further accelerate the electrons to reduce the space charge effects as well as to compress the electron bunch down to 3 ps. At the DC gun exit, the rms bunch length is $10 \mathrm{ps}$. With the proper adjustment to meet the above requirements, the RF phase of 20 degrees off-crest is chosen. The net energy gain is $1.5 \mathrm{MeV}$ and the rms bunch length is compressed from 10 ps before buncher to 3 ps after the buncher, as shown in Figure 3.

The $2^{\text {nd }}$ solenoid matches the beam optics into two accelerating structures. Each structure consists of a 9-cell L-band standing wave structure operating at an accelerating gradient of $10 \mathrm{MV} / \mathrm{m}$. The transverse phase spaces and longitudinal spectrum before and after the accelerating structures presented in Figure 4. It is shown that the divergence is smaller after $2^{\text {nd }}$ structure compared with the one before $1^{\text {st }}$ structure. However, their emittances are almost the same.
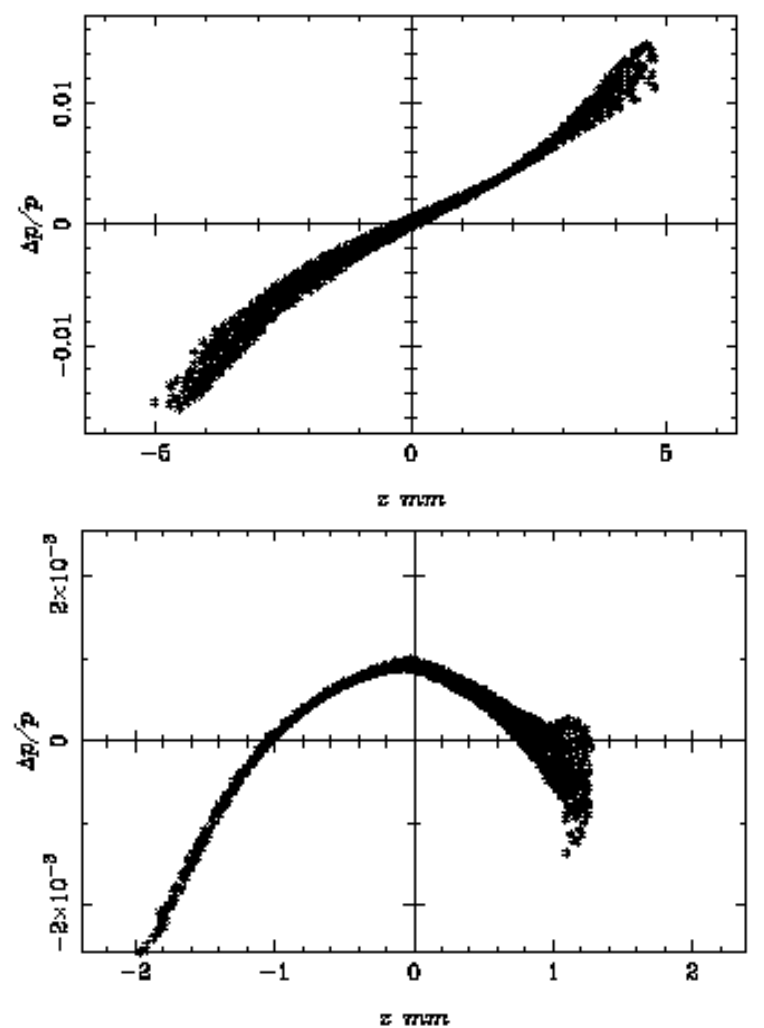

Figure 3: Longitudinal spectrum before and after buncher (trans. scale is from -0.002 to 0.002 in lower plot)
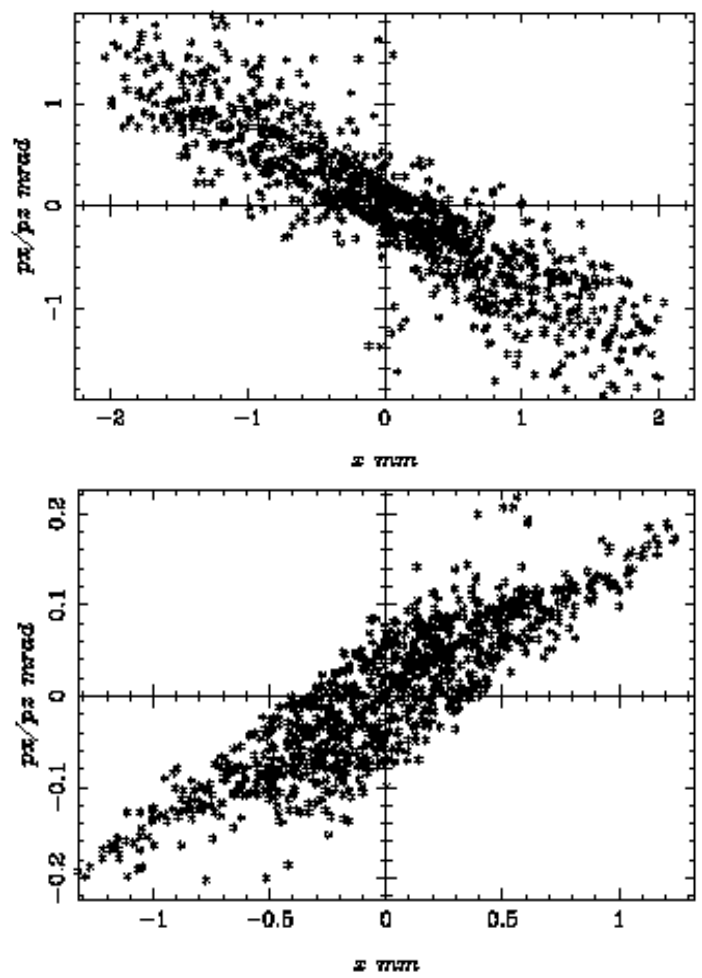

Figure 4: Transverse spectrum before and after accelerating structures 


\subsection{Beam Dynamics Simulations}

Beam dynamics studies were carried out from the cathode to the end of the injector using DESY developed computer code ASTRA [5]. Both space charge and its mirror charge from the cathode are included in the simulations. The initial longitudinal distribution is flattopped with $25 \mathrm{ps}$ on top and $2 \mathrm{ps}$ for both rise and fall edges. The uniform distribution is assumed initially with rms radius $1 \mathrm{~mm}$. For a bunch charge of $0.15 \mathrm{nC}$, the transverse emittance and beam size along the beam line are plotted in Figures 5. It is shown that, the transverse emittance and beam size were optimized at the end of beam line to be $0.9 \mathrm{~mm}-\mathrm{mrad}$ and $0.5 \mathrm{~mm}$, respectively.
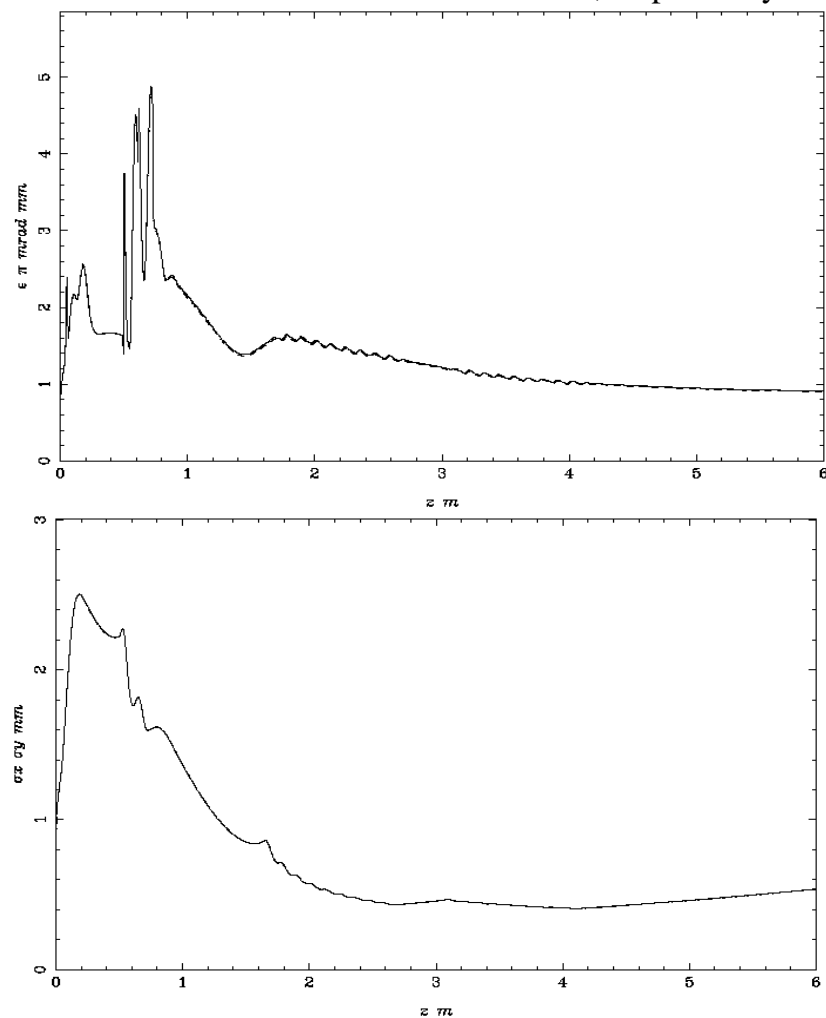

Figure 5: Trans. emittance and beam size

(Upper: $0-6 \mathrm{~m}$ in longitudinal and $0-5 \mu \mathrm{m}$ in transverse

Lower: $0-6 \mathrm{~m}$ in longitudinal and $0-3 \mathrm{~mm}$ in transverse)

The longitudinal emittance and bunch length are also calculated (Figure 6). It is shown that the longitudinal emittance and the rms bunch length are almost kept to be constant after $2^{\text {nd }}$ structure (exit of $2^{\text {nd }}$ structure is about at 4 meter in the plots), $18.5 \mathrm{~mm} . \mathrm{keV}$ and $0.95 \mathrm{~mm}$, respectively. The energy spread at the end of the injector is about $27 \mathrm{keV}$.

\section{SUMMARY AND OUTLOOK}

We have carried out preliminary beam dynamics simulation for a DC gun, conclude that the main PERL beam parameters can be met using a DC gun. The results are summarized in Table 1. Further studies are required to investigate the feasibility of DC gun.
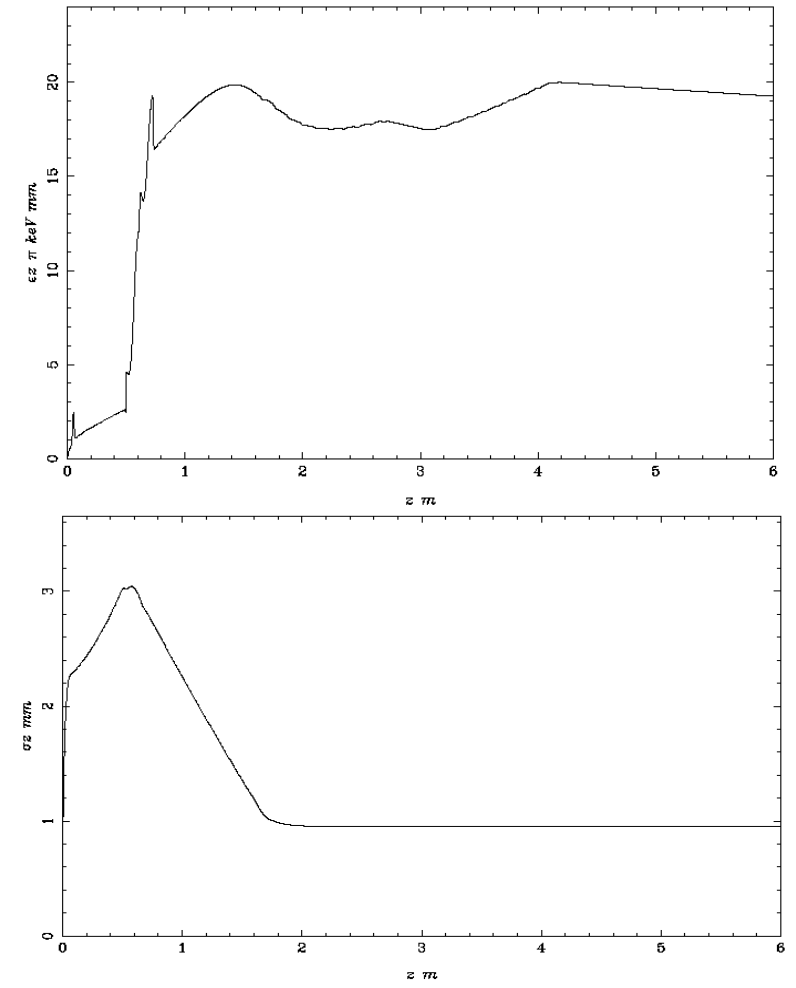

Figure 6: Longitudinal emittance and rms bunch length (Upper: 0-6m in longi. and 0-20mm.kev in trans. scale; Lower: $0-6 \mathrm{~m}$ in longi. and $0-3 \mathrm{~mm}$ in trans. scale)

Table 1: Comparison of the simulation results and the PERL requirements

\begin{tabular}{|l|c|c|}
\hline & Simulation & Requirement \\
\hline Energy $(\mathrm{MeV})$ & 23 & 25 \\
\hline Bunch charge $(\mathrm{nC})$ & 0.15 & 0.15 \\
\hline $\begin{array}{l}\text { Rms energy spread } \\
(\mathrm{keV})\end{array}$ & 27 & 25 \\
\hline $\begin{array}{l}\text { Rms bunch length } \\
\text { (mm) }\end{array}$ & 0.95 & 0.9 \\
\hline $\begin{array}{l}\text { Longitudinal emittance } \\
\text { (mm.keV) }\end{array}$ & 18.5 & - \\
\hline $\begin{array}{l}\text { Transverse emittance } \\
\text { (mm.mrad) }\end{array}$ & 0.9 & 1 \\
\hline
\end{tabular}

\section{ACKNOWLEDGEMENT}

Authors would like to thank Dr. T. Shaftan for his helpful discussion, comments and suggestions from other members of NSLS PERL study group

\section{REFERENCES}

[1] I.Ben-Zvi, et al., PERL upgrade for NSLS, this conference.

[2] X.J.Wang(ed.), Proceeding of workshop on PERL injetcor, BNL-52624, 2001.

[3] K.J. Kim, NIM A 285 (1989) 313.

[4] C.Sinclair, A $500 \mathrm{kV}$ photoemission electron gun for the CEBAF FEL, CEBAF-PR-91-035.

[5] K.Floettmann, Maual of ASTRA code, DESY, Germany 1999. 